\title{
Cultivo axênico das cianobactérias marinhas Aphanothece halophytica Frémy, 1933 e Chroococcus minutus (Kützing) Nägeli, 1849
}

Rodrigo de Siqueira Melo ${ }^{1,2}$, Maria Helena Campo Baeta Neves ${ }^{1}$ e Odara Ramôa Baptista ${ }^{1}$

Recebido em 24/04/2010. Aceito em 11/03/2011

\begin{abstract}
RESUMO
(Cultivo axênico das cianobactérias marinhas Aphanothece halophytica Frémy, 1933 e Chroococcus minutus (Kützing) Nägeli, 1849). O presente estudo demonstra o método utilizado para a obtenção do cultivo axênico das cianobactérias Aphanothece halophytica e Chroococcus minutus, obtidas das salinas do município de Arraial do Cabo - RJ. Nas salinas, esses organismos estão estruturados em mats microbianos, o que torna o seu isolamento mais difícil e, consequentemente, a obtenção de culturas axênicas. Desta forma, utilizando a combinação de técnicas de microscopia associado à utilização de azida sódica e fluoreto de sódio para inibição da cadeia transportadora de elétrons e da glicólise respectivamente, apenas os microorganismos que realizassem fotossíntese conseguiriam sobreviver. Durante o crescimento das cianobactérias, foram realizadas medições biométricas das características morfológicas para monitorar o desenvolvimento desses microorganismos em meio limitante. Ao final do período proposto de crescimento, observamos que a metodologia aplicada para o isolamento e obtenção das culturas axênicas foi eficaz como uma alternativa para cultivar as cianobactérias A. Halophytica e C. minutus.
\end{abstract}

Palavras-chave: azida sódica, fluoreto de sódio, morfoespécies, BG-11 e cultura unialgal

\begin{abstract}
(Axenic culture of the sea cyanobacterias Aphanothece halophytica Frémy, 1933 and Chroococcus minutus (Kützing) Nägeli, 1849). This work demonstrates the method used to obtain axenic culture of the cyanobacteria Aphanothece halophytica and Chroococcus minutes, both from salt flats in Arraial do Cabo municipality, Rio de Janeiro state. In salt flats, these organisms are structured microbial mats, which makes their isolation more difficult and consequently the establishment of axenic cultures. Thus, using a combination of microscopy techniques associated with the use of sodium azide and sodium fluoride for inhibition of the electron transport chain and glycolysis, respectively, only microorganisms that carry out photosynthesis could survive. During cyanobacteria growth, biometric measurements were performed to monitor the morphological development of these microorganisms in limiting medium. At the end of the proposed growth period, we observed that the methodology applied for isolation and obtaining axenic cultures was effective as an alternative to grow the cyanobacteria A. halophytica and C. minutus.
\end{abstract}

Key words: sodic azide, sodium fluoreto, morphospecies, BG-11 and unialgal culture

\section{Introdução}

As cianobactérias são organismos fotossintetizantes procarióticos e filogeneticamente do grupo das bactérias gram-negativas, com distribuição cosmopolita, variando de ambientes extremos como fontes termais à regiões Árticas e Antarticas (Sinha \& Häder 2008). Os "algal mats" são esteiras microbianas presentes em ambientes com extrema salinidade, onde a incidência luminosa determina as posições verticais estratificadas dos grupos funcionais de microorganismos neste ambiente (Ladakis et al. 2006).
Segundo Badger et al. (2006), nos ambientes hipersalinos, as cianobactérias possuem um função chave na produção de uma mucilagem, auxiliando a estruturação dos algal mats. A mobilidade das cianobactérias, em associação com uma notável excreção de polissacarídeos extracelulares, possibilita os organismos a se distribuírem em diferentes tipos de sedimentos (Rios et al. 2004).

As cianobactérias são organismos halotolerantes por possuírem mecanismos específicos que ajustam intracelularmente o status osmótico (Apse \& Blumwald 2002). A. halophytica e C. minutus podem desenvolver-se em

1 Instituto de Estudos do Mar Alte Paulo Moreira, Departamento de Oceanografia, Arraial do Cabo, RJ, Brasil

2 Autor para correspondência: rodrigosqm@gmail.com 
condições de extrema alcalinidade através da regulação de canais de $\mathrm{Na}+$ / H+ e tolerância de 0.25 à $3.0 \mathrm{M}$ de cloreto de sódio $(\mathrm{NaCl})$ devido à produção de moléculas osmoprotetoras como glicina betaina, trealose e glicerol (Laloknam et al. 2006).

Nos mats, essas espécies de cianobactérias possuem a capacidade de sobreviver em condições de baixa concentração nutricional, intensa variação luminosa, longos períodos de dessecação, alta produção de exopolissacrídeos, limitação da difusão de $\mathrm{O}_{2}$ e $\mathrm{CO}_{2}$ e variabilidade de temperatura e salinidade (Badger et al. 2006). Como consequência, as cianobactérias formam agregados onde bactérias e outros organismos estão firmemente aderidos, respresentando uma grande dificuldade para obtenção de culturas unialgais e axênicas (Vázquez-Martínez et al. 2004). Desta forma, diversos métodos para obtenção de culturas axênicas de cianobactérias têm sido sugeridos (Choi et al. 2002). Em alguns métodos descritos na literatura, como por exemplo o uso de antibióticos seletivos, provou ser ineficaz na presença de mucilagem produzida pela cianobactérias, por abrigarem e protegerem as bactérias dos antibióticos (Cho et al. 2002).

Desta forma, o presente trabalho teve como objetivo coletar, isolar e obter cultivo axênico das espécies de cianobactérias A. halophytica e C. minutus, obtidas a partir dos algal mats das salinas de Arraial do Cabo (RJ).

\section{Material e métodos}

\section{Coleta dos algal mats}

As cianobactérias unicelulares e halotolerantes Chroococcus minutus (Kützing) Nägeli 1849 e Aphanothece halophytica Frémy 1933 foram isoladas a partir de amostras de "microbial mats", coletados em uma profundidade de $0-5 \mathrm{~cm}$ nos cristalizadores das salinas do município de Arraial do Cabo (22 $56^{\prime} 57$ S e $42^{\circ} 04^{\prime} 19$ W, Estado do Rio de Janeiro - RJ - Brasil). A amostra foi transportada para o Laboratório de Biofilme do Instituto de Estudos do Mar Almirante Paulo Moreira - IEAPM (Marinha do Brasil) e mantida sob refrigeração a $15^{\circ} \mathrm{C}$. Posteriormente, a amostra foi avaliada por meio de lupa e de microscópio óptico com o propósito de determinar a camada no "algal mats" onde se localizavam as cianobactérias de interesse com vista à sua remoção para a realização do isolamento, sendo utilizado o sistema de classificação taxonômica de Komárek \& Anagnotidis (1999).

\section{Elaboração do meio de cultura}

O meio de cultura líquido foi preparado dissolvendo todos os componentes mencionados na Tabela 01 em água do mar pré-tratada através de filtração e, posteriormente, esterilizada em autoclave a $120^{\circ} \mathrm{C}$ durante $20 \mathrm{~min}$, possuindo $\mathrm{pH}$ final de 7.1 e salinidade de 50 (5,0 \%) após seu resfriamento.

O meio de cultura sólido foi constituído dissolvendo agar a 1,5\% no meio BG-11 modificado pela adição de
Tabela 1. Composição do meio de cultura BG-11

\begin{tabular}{lc}
\hline Componentes & Concentração g/L \\
\hline $\mathrm{NaNO}_{3}$ & 1,5 \\
$\mathrm{NaCl}$ & 15 \\
$\mathrm{~K}_{2} \mathrm{HPO}_{4}$ & 0,04 \\
$\mathrm{MaSO}_{4} \cdot 7 \mathrm{H}_{2} \mathrm{O}$ & 0,075 \\
$\mathrm{CaCl}_{2} \cdot 2 \mathrm{H}_{2} \mathrm{O}$ & 0,036 \\
$\mathrm{Na}_{2} \mathrm{CO}_{3}$ & 0,02 \\
Ácido cítrico & 0,006 \\
$\mathrm{Citrato} \mathrm{férrico} \mathrm{de} \mathrm{amônio}$ & 0,006 \\
EDTA & 0,001 \\
Micronutrientes $(*)$ & $1 \mathrm{~mL} / \mathrm{L}$ \\
\hline
\end{tabular}

* $\mathrm{H}_{3} \mathrm{BO}_{3}: 2,86 \mathrm{~g}$ /litro; $\mathrm{MnCl}_{2} \cdot 4 \mathrm{H}_{2} \mathrm{O}: 1,81 \mathrm{~g} /$ litro; $\mathrm{ZnSO}_{4} \cdot 7 \mathrm{H}_{2} \mathrm{O}: 0,222 \mathrm{~g} /$ litro; $\mathrm{Na}_{2} \mathrm{MoO}_{4} \cdot 2 \mathrm{H}_{2} \mathrm{O}: 0,39 \mathrm{~g} / \mathrm{litro} ; \mathrm{CuSO}_{4} \cdot 5 \mathrm{H}_{2} \mathrm{O}: 0,079 \mathrm{~g} / \mathrm{litro} ; \mathrm{Co}\left(\mathrm{NO}_{3}\right)_{2} \cdot 6 \mathrm{H}_{2} \mathrm{O}$ : $0,0494 \mathrm{~g} /$ litro.

bicarbonato $\left(\mathrm{HCO}_{3}^{-}\right)$na concentração final de $3 \mathrm{mM}$. O agar foi preparado com o dobro da concentração desejada (3\%) e cortado em pequenos blocos, que foram lavados em água destilada durante 96 horas com o objetivo de eliminar impurezas que poderiam inibir o crescimento em gelose. Os blocos de agar foram, então, aquecidos e misturados na proporção de 1:1 com o meio BG-11 modificado contendo o dobro da concentração final dos seus sais constituintes.

Após a separação da camada de cianobactérias nos mats utilizando espátula, essas foram colocadas em erlenmeyer de $250 \mathrm{~mL}$ de capacidade contendo $100 \mathrm{~mL}$ do meio de cultura sintético BG-11 (Stanier et al. 1971), cuja composição encontra-se descrita na Tabela 01. Estas foram inoculadas pelo período de 15 dias e, posteriormente, inoculadas em meio BG-11 sólido para obtenção de culturas unialgais.

\section{Condições de cultivo}

Cepas, contendo $1 \times 10^{4}$ cels $/ \mathrm{mL}$, foram colocadas em tubos de ensaio, contendo $10 \mathrm{~mL}$ de meio de cultura BG-11, inclinados, sob iluminação constante $(20 \mu$ mols de fótons.m $\left.{ }^{2} \cdot \mathrm{s}^{-1}\right)$ provida por lâmpadas fluorescentes e incubados na temperatura de $28 \pm 2{ }^{\circ} \mathrm{C}$ durante 15 dias. Após esse período, amostras foram transferidas para placas de petri, contendo o meio BG-11 sólido, e incubadas a $25 \pm 2{ }^{\circ} \mathrm{C}$ durante 10 dias. Repicagens sucessivas foram feitas com alça de platina esterilizada para novas placas com o propósito de separar as colônias que apresentavam crescimento conspícuo, visando à obtenção de culturas unialgais. Essas culturas foram posteriormente cultivadas em erlenmeyers de $500 \mathrm{~mL}$, contendo $200 \mathrm{~mL}$ de meio de cultura líquido a $28 \pm 2{ }^{\circ} \mathrm{C}$.

Obtenção de cultura axênica

Para obtenção de culturas axênicas de A. halophytica e C. minutus, foram recolocadas em tubos de ensaio, contendo 10 $\mathrm{mL}$ de meio líquido. Após acompanhamento do crescimento em microscopia óptica, as células foram precipitadas por 
centrifugação a $5000 \mathrm{x} g$ por 10 minutos e ressuspensas em tubos de ensaio, contendo $10 \mathrm{~mL}$ de meio líquido, acrescido de um inibidor da cadeia respiratória (azida sódica na concentração final de $5 \mathrm{mM}$ ) e um inibidor da glicólise (fluoreto de sódio na concentração final de $50 \mathrm{mM}$ ), de modo que apenas o crescimento fotoautotrófico fosse permitido. Após sete dias de crescimento, na presença dos inibidores, as cianobactérias foram transferidas para meio sólido e, após subcultivos em meio sólido, foi possível obter culturas axênicas, mantidas a $28 \pm 2{ }^{\circ} \mathrm{C}$ sob iluminação constante (20 $\mu$ mols de fótons. $\left.\mathrm{m}^{-2} \cdot \mathrm{s}^{-1}\right)$ (Magalhães 1999).

\section{Resultados e discussão}

Segundo Neves \& Casarin (1990), os algal mats das salinas de Arraial do Cabo - RJ, onde as cianobactérias foram coletadas, são constituídos por uma mistura de espécies organizadas em diferentes camadas junto com pequenos depósitos minerais em um gradiente crescente de salinidade. Essa complexidade estrutural dos mats implicou na necessidade de combinar técnicas diferentes de microscopia e a utilização de lupa, microscópico ótico e de epifluorescência, para analisar in situ, os diferentes componentes algais dos mats sem perturbar a organização do sistema. Desta forma, conseguimos isolar e obter culturas axênicas de $A$. halophytica e C. minutus.

A diversidade estrutural das cianobactérias é considerável e, por esta razão, os ficólogos puderam desenvolver os tratados taxonômicos do grupo baseado unicamente em propriedades estruturais, determinadas através da observação microscópica (Geitler 1932). Entretanto, Komárková et al. (2010) utilizando técnicas moleculares, como a análise da subunidade $16 \mathrm{~S}$ do $\mathrm{rRNA}$, demonstrou a variabilidade morfológica das espécies do gênero Chroococcus e a correlação com a análise filogenética.

De acordo com Komárek \& Anagnostidis (1999), a ordem Chroococcales compreende cerca de 11 famílias com 90 gêneros, incluindo formas unicelulares e coloniais. Os planos de divisão celular, a morfologia, tamanho das células e colônias, presença de aerótopos, característica do envelope mucilaginoso e habitat são fundamentais para caracterização de diferentes grupos de cianobactérias da ordem Chroococcales.

A classificação das Chroococcales é controversa, e mesmo as identificações genéricas requerem uma escolha entre dois tratamentos taxonômicos muito diferentes na ordem (Drouet \& Daily, 1956). Os autores usam o plano ou os planos de divisões celulares como um caráter determinante preliminar. Essa propriedade é correlacionada com a forma celular, sendo dependente das condições de cultivo e do estágio de crescimento. Deve-se enfatizar que os planos de divisões celulares não podem sempre ser determinados com certeza pela observação microscópica simples porque, em muitas culturas, a segregação das células-filhas ocorre logo após a conclusão da divisão celular (Stanier et al., 1971).
Segundo Komárek \& Anagnostidis (1999), o sistema de classificação das cianobactérias unicelulares e coloniais está baseado na combinação de vários fatores por serem considerados geneticamente estáveis:

- polaridade das células e colônias são classificadas como um sinal diacrítico entre as famílias simples e mais complexas;

- o tipo da divisão celular (modificação da fissão binária e múltipla) é constante, de tal modo que pode servir para a delimitação das famílias. Dois ou diversos tipos da divisão celular podem ocorrer em diversas famílias, e a combinação de tipos de divisão é aceita como critério de definição da família.

O modo de vida unicelular ou colonial é um critério de diferenciação dos gêneros dentro das famílias. A forma e estrutura das colônias, formação de vários tipos de mucilagem, bainhas e a posição celular na colônia são critérios particularmente importantes.

O gênero Chroococcus pertence à família Chroococcace$a e$, sendo caracterizado por possuir suas células ou grupo de células rodeadas por envelope mucilaginoso, raramente solitárias (estágio inicial), células irregulares ou mais ou menos esféricas, colônias com bainha difluente, geralmente com poucas células ( 2 - 16), raramente formando aglomerações, nunca como tricoma ou uma organização regular. As células formam colônias multicelulares organizadas de 2 a 8 células. Células com coloração esverdeada, azul-esverdeada, verde-oliva, amarelada, alaranjada, avermelhada ou violeta, com conteúdo homogêneo ou granular. Plano de divisão celular em 3 planos em sucessivas gerações (usualmente perpendicular a algum outro plano).

A espécie Chroococcus minutus pode ser taxonomicamente diferenciada analisando a largura e aspecto da mucilagem, a largura e comprimento de cada célula, plano de divisão e conteúdo celular. Nos mats microbianos das salinas de Arraial do Cabo, suas colônias são caracterizadas possuindo de 2 - 4 células, com dimensões de 7,2 - 8,5 $\mu \mathrm{m}$ de comprimento e 7,5 - 9,8 $\mu \mathrm{m}$ de largura para cada indivíduo $(\mathrm{n}=50)$ e, considerando-se a bainha, 10,2 - 11,0 $\mu \mathrm{m}$ de comprimento e 11,3-14,0 $\mu$ m de largura $(\mathrm{n}=50$ ) ( ura 1a). Quando observadas sob microscopia óptica, apresentam conteúdo celular azul - esverdeado, homogêneo com bainha hialina não lamelada. Em cultura, $C$. minutus foi encontrado na forma de células isoladas ou em colônias de 2 a 4 células, com dimensões de 5,2 - 7,6 $\mu \mathrm{m}$ de comprimento e 5,4 - 11,0 $\mu \mathrm{m}$ de largura para cada indivíduo $(\mathrm{n}=50)$ e, considerando-se a bainha, 7,8 - 10,1 $\mu \mathrm{m}$ de comprimento e $8,0-13,5 \mu \mathrm{m}$ de largura (Figura $1 \mathrm{~b}$ e $1 \mathrm{c}$ ). Quando observadas sob microscopia de epiflurescência, apresentam conteúdo celular azul - esverdeado, homogêneo e, quando em divisão, heterogêneo, inseridas em bainha hialina não lamelada (Figura 1d).

Silva et al. (2004) relatam a presença de C. minutus em sedimento da Lagoa Hipersalina de Pitanguinha (RJ) e nas construções estromatolíticas da Lagoa Hipersalina Verme- 


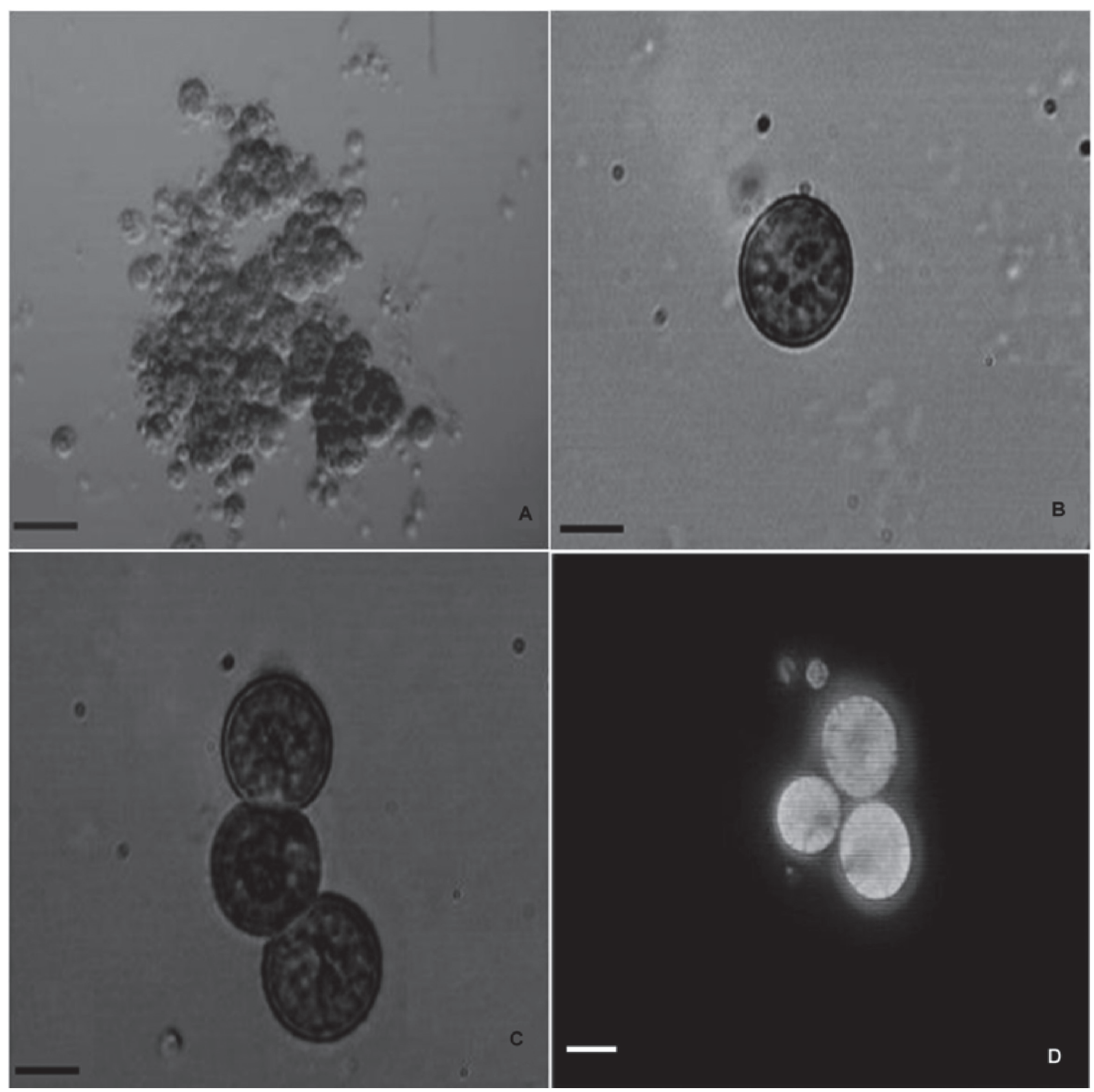

Figura 1. Chroococcus minutus em cultura: (A) aspecto colonial em cultura; (B) células solitárias; (C) células em divisão binária. (D) cultura monoespecífica e axênica - microscopia de epiflurescência. Barra $=10 \mu \mathrm{m}$,

lha (RJ). Díez et al. (2007) encontraram C. minutus na Ilha Eron (Grande Barreira de Recifes de Coral - Austrália) e nas condições morfométricas semelhantes aos espécimes constatados nas salinas do Município de Arraial do Cabo (RJ).

A espécie Aphanothece halophytica pertence à ordem Chroococcales, família Synechococcaceae e sub-família Aphanothecoideae. Essa espécie é taxonomicamente diferenciada, analisando a largura e aspecto da mucilagem, a morfologia celular (células arredondadas no ápice ou ovais), largura, comprimento, plano de divisão (plano perpendicular ao longo de seu eixo) e conteúdo celular. Nos mats microbianos das salinas, as colônias foram observadas possuindo células esféricas, com 6,1 - 8,9 $\mu \mathrm{m}(\mathrm{n}=50)$ de diâmetro. Conteúdo celular azul - esverdeado, homogêneo, inseridas em mucilagem não lamelada, hialina e difluente (Figura 2). Em cultura, A. halophytica foi encontrada em células isoladas, esféricas sem bainha 4,0 - 7,6 $\mu \mathrm{m}$ de diâmetro, com bainha 7,5 -9,5 $\mu \mathrm{m}$ de diâmetro $(\mathrm{n}=50)$. Conteúdo celular azul - esverdeado, homogêneo, inseridas em mucilagem hialina não lamelada. Nas mesmas condições morfológicas encontradas nas salinas de Arraial do Cabo (RJ), Silva et al., (2006) relatam A. halophytica em sedimento da Lagoa Hipersalina de Pitanguinha (RJ), em estromatólitos da Lagoa Pernambuco - Cabo Frio (RJ) e em mats microbianos das 


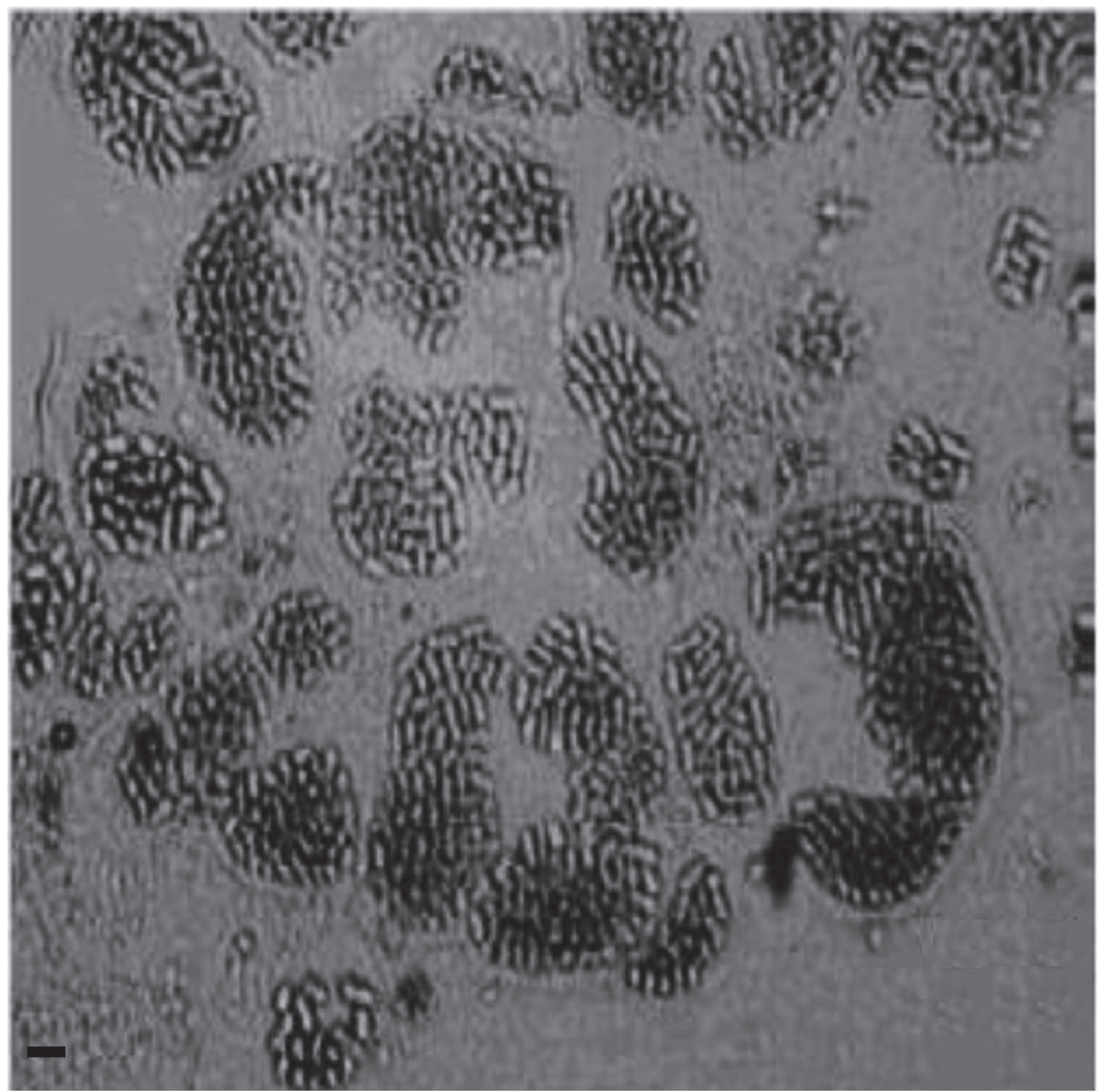

Figura 2. Aspecto colonial de A. halophytica nos mats microbianos das salinas de Arraial do Cabo - RJ. Barra $=10 \mu \mathrm{m}$.

salinas de Araruama. Oren et al., (1995) encontraram esta espécie em mats microbianos nas poças de maré em Israel.

O mecanismo de concentração de carbono inorgânico está baseado no transporte $\mathrm{CO}_{2}$ e/ou $\mathrm{HCO}_{3}$ pela membrana do tilacóide. Posteriormente, o carbono na forma de bicarbonato é transportado aos carboxiossomos, onde a enzima anidrase carbônica catalisa uma reação em que transforma bicarbonato em dióxido de carbono. Esse mecanismo de indução no influxo de $\mathrm{HCO}_{3}^{-}$ocorre naturalmente como uma adaptação das cianobactérias ao baixo nível de carbono inorgânico dissolvido no meio (Espie \& Kandasamy, 1992). Portanto, em laboratório, o meio de cultura BG-11, utilizado no cultivo de A. halophytica e C. minutus, foi modificado pela adição de $\mathrm{HCO}_{3}^{-}$para aumentar a fonte de carbono inorgânico. Com o objetivo de padronizar a fase para análise das características morfométricas (diâmetro, largura, comprimento celular e espessura da mucilagem extracelular), foram acompanhadas as curvas de crescimento das duas espécies de cianobactérias estudadas: A. halophytica e C. minutus. Para essa padronização, as espécies foram adaptadas às condições de cultivo durante 48 horas e, posteriormente, foram realizadas as contagens para a elaboração da curva de crescimento durante 120 horas. 
A fase lag de crescimento é caracterizada por não haver aumento do número de células. Entretanto, nesse período, as células estão metabolicamente ativas, reparando possíveis danos celulares e sintetizando enzimas. A fase lag é seguida por um período de crescimento exponencial (fase $\log$ ), em que observamos a maior taxa de crescimento celular para A. halophytica em 36 horas e em 48 horas para C. minutus, sendo que o crescimento perdurou até $50 \mathrm{~h}$ em ambas as culturas de cianobactérias (Figura 3). Desta forma, do período de $50 \mathrm{~h}$ a $96 \mathrm{~h}$ observação a fase de desaceleração do crescimento. Essa multiplicação celular cessa quando os nutrientes são consumidos ou quando os produtos metabólicos se acumulam em quantidades suficientes para que o crescimento seja interrompido (Pelczar et al., 1997). Nesse caso, essa fase de crescimento (fase estacionária), em que não ocorre mais um aumento no número de células, pode ser observada a partir de 96 horas. Em ambas as culturas, não foi observada a fase de declínio do número de células durante o tempo de acompanhamento do cultivo celular (Pelczar et al., 1997). O período de observação do cultivo, isto é, 168 horas, foi suficiente para mostrar o comportamento do crescimento de A. halophytica e C. minutus nas condições estabelecidas em laboratório. A curva de crescimento de A. halophytica e C. minutus foi a mesma observada por Goldbeck et al. (2006). Entretanto, em meio BG-11, Aphanothece microscopica (Nägeli), atinge a fase estacionária em $50 \mathrm{~h}$ do seu desenvolvimento em cultura. Fernandes et al. (1993), realizaram estudos de fixação de nitrogênio e estresse osmótico com cianobactérias, em que as curvas de crescimento obtidas foram realizadas durante um período de 120 horas. Da mesma forma, Bonilla et al. (1990), em estudos realizados sobre o requerimento de boro por cianobactérias, demonstram os dados do desenvolvimento celular em curvas de crescimento realizadas em 120 horas de observação.

O presente estudo centrou-se na aplicação de metodologia de isolamento e obtenção de cultivo axênico de duas espécies de cianobactérias A. halophytica e C. minutus obtidas dos algal mats de ambiente hipersalino. Os resultados demonstram que o método utilizado para o isolamento e as condições de cultivo foram satisfatórias para obtenção do cultivo proposto. Dessa forma, perspectivas de contínuos estudos para o entendimento de suas características fisiológicas e bioquímicas se fazem necessários, visto a sua aplicação biotecnológica nos diferentes ramos industriais.

\section{Referências Bibliográficas}

Apse, M.P. \& Blumwald, E. 2002. Engineering Salt Tolerance in Plants. Curr. Opin. Plant Biotechnol. 13: 146-150.

Badger, M.R.; Price, G.D. \& Long, B.M. et al. 2006. The Environmental Plasticity and Ecological Genomics of the Cyanobacterial $\mathrm{CO}_{2}$ Concentrating Mechanism. Journal of Experimental Botany 57: 249-265.

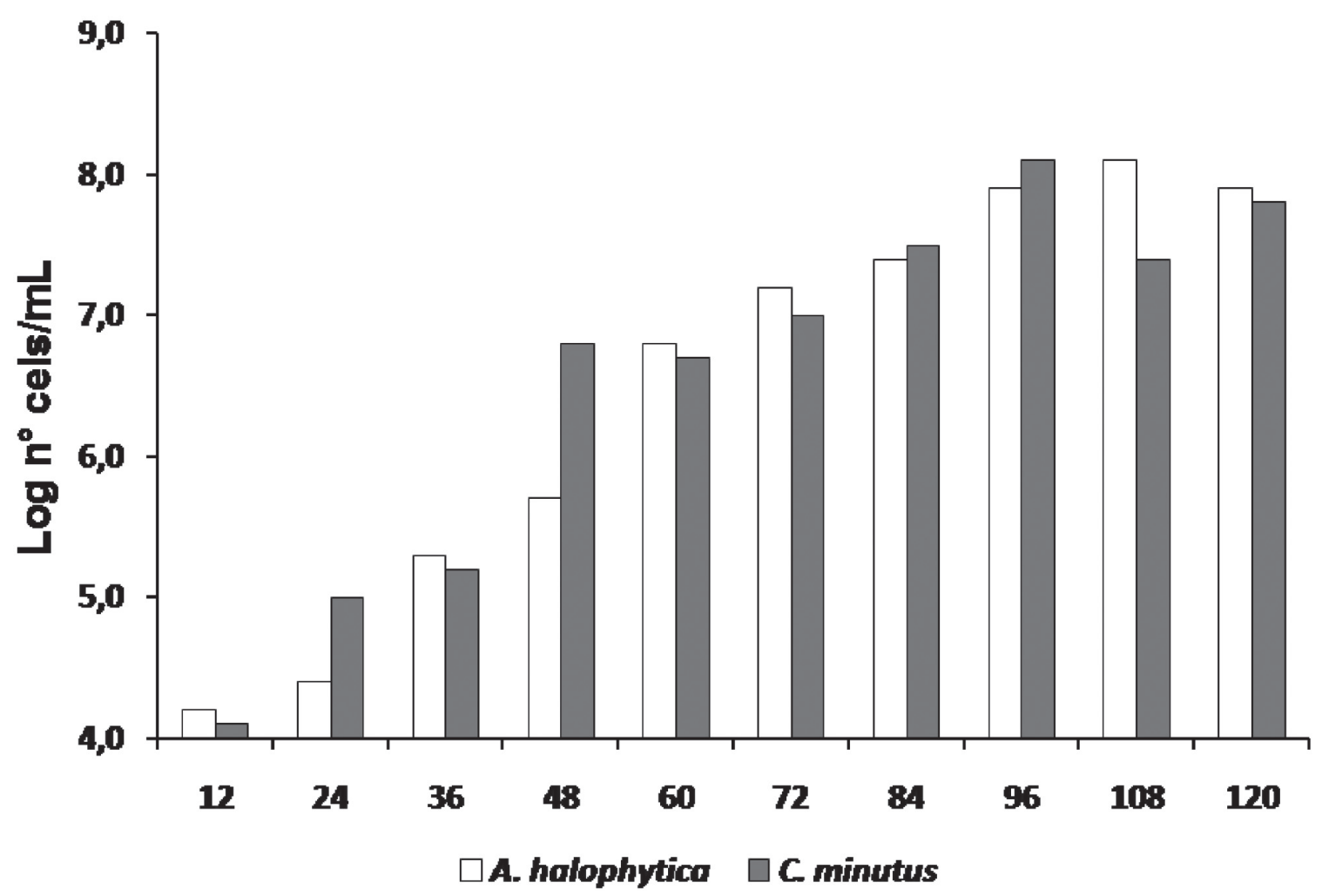

Figura 3. Curva de crescimento de A. halophytica e C. minutus nas condições de manutenção da cultura (meio BG-11 modificado, $28 \pm 2{ }^{\circ} \mathrm{C} \mathrm{e} 20 \mu$ mol de fótons. $\left.\mathrm{m}^{-2} \cdot \mathrm{s}^{-1}\right)$. 
Bonilla, L.; Garcia-Gonzalez, M. \& Mateo, P. 1990. Boron Requirement in cyanobacterial Its Possible Role in the Early Evolution of Photosynthetic Organisms. Plant Physiol 94: 1554-1560.

Cho, J.Y.; Choi, J.S.; Kong, I.S.; Park, S.I.; Kerr, R.G. \& Hong, Y.K. 2002. A Procedure for Axenic Isolation of the Marine Microalga Isochrysis galbana from Heavily Contaminated Mass Cultures. Journal of Applied Phycology 14: 385-390.

Choi, J.S.; Cho, J.; Jin, L.G.; Jin, H.J. \& Hong, Y.K. 2002. Procedures for the Axenic Isolation of Conchocelis and Monospores from the Red Seaweed Porphyra yezoensis. Journal of Applied Phycology 14: 115-121.

Díez, B.; Bauer, K.; Bergman, B. 2007. Epilithic Cyanobacterial Communities of a Marine Tropical Beach Rock (Heron Island, Great Barrier Reef): Diversity and Diazotrophy. Applied and Environmental Microbiology 73(11): 3656-3668.

Drouet, F.; Daily, W.A. 1956. Revision of the coccoid Myxophyceae. Butler Univ. Bot. Stud. 12: 1-122.

Espie, G.S.; Kandasamy, R.A. 1992. $\mathrm{Na}^{+}$Independent $\mathrm{HCO}_{3}^{-}$Transport and Accumulation in the Cyanobacterium Synechococcus UTEX 6251. Plant Physiol 98: 560-568.

Fernandes, T.A.; Lyer, V. \& Apte, S.K. 1993. Differential Responses of Nitrogen-fixing Cyanobacteria to Salinity and Osmotic Stresses. Applied and Environmental Microbiology 59: 899-904.

Geitler, L. 1932. Cyanophyceae. In R. Kolkwitz (ed.), Kryptogamenfiora von Deutschland, Osterreich und der Schweiz. Akademische Verlagsgesellschaft, Leipzig 14: 196p.

Goldbeck, R.; Silva, A.G.; Jacques, A.C.; Cogo, S.L.; Mitterer, M.L. \& Queiroz, M.I. 2006. Relação entre o nitrogênio total e não-proteíco na cianobactéria Aphanothece microscopica Nägeli. Vetor 16(1/2): 47-53.

Komárková, J.; Jezberová, J.; Komárek, O. \& Zapomelová, E. 2010. Variability of Chroococcus (Cyanobacteria) morphospecies with regard to phylogenetic relationships. Hydrobiologia 639: 69-83.

Komárek, J. \& Anagnostidis, K. (1999). Subwasserflora Von mitteleuropa band 19/1: Cyanoprocaryota 1. Teil: Chroococcales. Gustav Fisher. Stuttgart. 548p.

Ladakis, M; Dassenakis, M. \& Pantazidou, A. 2006. Nitrogen and Phosphorus in Coastal Sediments covered by Cyanobacteria Mats. J. Soils \& Sediments 6(1): 46-54.
Laloknam, S.; Tanaka, K.; Buaboocha, T.; Waditee, R.; Incharoensakdi, A.; Hibino, T.; Tanaka, Y. \& Takabe, T. 2006. Halotolerant Cyanobacterium Aphanothece halophytica Contains a Bataine Transporter Active at Alkaline $\mathrm{pH}$ and High Salinity. Applied and Environmental Microbiology 72: 6018-6026.

Magalhães, C.C.P. 1999. Respostas fisiológicas e fotossintéticas da cianobactéria Synechocystis aquatilisf. aquatilis a altas concentrações de zinco. Tese de Mestrado em Bioquímica, Instituto de Química. Rio de Janeiro, UFRJ.

Neves, M.H.C.B. \& Casarin, A. 1990. Cianofíceas das salinas de Cabo Frio - Brasil. Acta Biologica Leopoldensia 12: 99-123.

Oren, A.; Kühl, M. \& Karsten, U. 1995. An endoevaporitic microbial mat within a gypsum crust: zonation of phototrophs, photopigments, and light penetration. Marine Ecology Progress Series 128: 151-159.

Pelczar, M.J.; Chan, E.E.C.S. \& Krieg, N.R. 1997. Microbiologia: conceitos e aplicações. Makson Books, 2 ed. $01-524$.

Rios, A.L.; Ascaso, C.; Wierzchos, J.; Fernandez-Valiente, E. \& Quesada, A. 2004. Microstructural Characterization of Cyanobacterial Mats from the McMurdo Ice Shelf, Antarctica. Applied and Environmental Microbiology 70: 569-580.

Stanier, R.Y.; Kunisawa, R.; Mandel, M. \& Cohen-Bazire, G. 1971. Purification and Properties of Unicellular Blue-Green Algae (Order Chroococcales). Bacterological Reviews 35: 171-205.

Silva, L.H.S.; Damazio, C.M. \& Iespa, A.A.C. 2004. Identificação de Cianobactérias em Sedimentos da Lagoa Pitanguinha, Estado do Rio de Janeiro, Brasil. Anuário do Instituto de Geociências - UFRJ, 28(1): 92-100.

Silva, L.H.S.; Iespa, A.A.C.; Damazio, C.M. 2006. Trombólitos e Cianobactérias da Lagoa Pernambuco, Holoceno do Rio de Janeiro, Brasil. Revista de Biologia e Ciências da Terra 6(2): 243-250.

Sinha, R.P.; Häder, D. 2008. UV-protectants in Cyanobacteria. Plant Science 174: 278-289.

Vázquez-Martínez, G.; Rodriguez, M.H.; Hernández-Hernández, F. \& Ibarrab, J.E. 2004. Strategy to obtain axenic cultures from field-collected samples of the cyanobacterium Phormidium animalis. Journal of Microbiological Methods 57: 115-121. 